\title{
Computation of Dancoff Factors for Fuel Elements Incorporating Randomly Packed TRISO Particles
}

J. L. Kloosterman

A. M. Ougouag

January 2005

Idaho National Engineering and Environmental Laboratory Bechtel BWXT Idaho, LLC 


\title{
Computation of Dancoff Factors for Fuel Elements Incorporating Randomly Packed TRISO Particles
}

\author{
Jan L. Kloosterman* \\ Abderrafi M. Ougouag
}

January 2005

Idaho National Engineering and Environmental Laboratory Idaho Falls, Idaho 83415

Prepared for the

U.S. Department of Energy Office of Nuclear Energy Under DOE Idaho Operations Office

Contract DE-AC07-99ID13727 


\begin{abstract}
A new method for estimating the Dancoff factors in pebble beds has been developed and implemented within two computer codes. The first of these codes, INTRAPEB, is used to compute Dancoff factors for individual pebbles taking into account the random packing of TRISO particles within the fuel zone of the pebble and explicitly accounting for the finite geometry of the fuel kernels. The second code, PEBDAN, is used to compute the pebble-to-pebble contribution to the overall Dancoff factor. The latter code also accounts for the finite size of the reactor vessel and for the proximity of reflectors, as well as for fluctuations in the pebble packing density that naturally arises in pebble beds.
\end{abstract}




\section{EXECUTIVE SUMMARY}

The task reported on in this document was initially meant to investigate the methods available in the literature for accounting for the randomness of TRISO particle packing when computing Dancoff factors in doubly heterogeneous pebble beds. It was planned then to either determine which method is fully applicable to the design of the pebble bed version of the Next Generation Nuclear Plant (NGNP) or to produce a method that correctly accounts for the random packing of TRISO particles (this is referred to below as the infinite-medium or intra-particle Dancoff factor contribution). It was planned to retain the best method from the literature for incorporating the pebble-to-pebble effects on the overall Dancoff factor. However, during the course of this study it was determined that even the most widely accepted pebble-to-pebble method was not acceptable for the case of the NGNP, and a fully rigorous method (and code) was developed. These developments are summarized here.

Infinite-medium Dancoff factors are calculated for spherical kernels with a stochastic packing as used in High Temperature Reactors, and compared with four methods from the literature. The latter assume either infinitely small kernels with a random distribution, or finite kernels with some restrictive assumptions. In the new method developed in this work (and presented here for the first time), we calculate the infinite-medium Dancoff factor by numerically integrating the Dancoff factor of two adjacent kernels over their surfaces and respective positions. It turns out that for practical pebble-bed fuel designs all four methods give results accurate within $2 \%$, but that larger deviations are obtained for extreme cases (either at high or low dilution). It follows that whereas all methods are applicable to the currently contemplated design of the pebble for the NGNP, they are not equally applicable to possible alternate designs. Furthermore, the current study points to the likelihood of serious inadequacy of the methods currently in use in the literature and in computer codes developed elsewhere for the design of the prismatic version of the NGNP, in which substantially higher packing fractions of TRISO particles are contemplated. The method developed here, for the pebble case, should be applicable to the prismatic design case, with the appropriate modifications. The methods developed in this project are summarized in the next two paragraphs.

The first method pertains to the computation of the contribution of the "within-pebble" features to the overall doubly heterogeneous Dancoff factor. For this, a Monte-Carlo program called INTRAPEB was written that calculates the average value and the space-dependence of the Dancoff factor of one single fuel pebble. The code also computes the angular distribution of neutrons escaping the pebble. For the Dancoff factor, the analytical results from the literature agree very well with ours at TRISO packing values typical of the currently planned design of the pebble bed NGNP fuel. However, for a cubic packing of particles, as is usually modeled in MCNP calculations, a 10\% larger Dancoff factor is found, which might explain some differences in resonance absorption reported in the literature. In the nominal random packing case, the angular distribution of neutrons escaping from the moderator zone of a pebble predicted in this work is much more forwardly peaked than the cosine angular distribution assumed in analytical methods.

The second method developed in this work pertains to the contribution to the doubly heterogeneous Dancoff factor that arises from the presence of multiple pebbles (i.e., the inter-pebble and pebble-topebble effects). For this, a second program called PEBDAN was written that calculates the average value and the space dependence of the inter-pebble Dancoff factor (the probability that a neutron escaping from the fuel zone of a pebble crosses a fuel particle in another pebble) and the pebble-pebble Dancoff factor (the probability that a neutron escaping from the fuel zone of a pebble crosses the fuel zone of another pebble). In this program, the coordinates of the pebbles in a randomly packed bed are determined, after which the Dancoff factors are calculated by a Monte Carlo ray-tracing method. 
In order to determine the positions of the pebbles, a random placing of pebbles in the vat of the reactor is assumed, and then packing is allowed until no room to move is available. This procedure generates a porosity distribution for the pebble bed (or conversely a density or packing distribution). The distributions generated in the PEBDAN code were compared with experimental values. The radial porosity profile of the packed bed shows less pronounced peaks and a slightly larger average value than those experimental results. However, the experimental results are for a vat diameter to sphere diameter ratio that is much larger than that of the NGNP pebble bed, and hence a direct comparison is not fully valid. A more recently developed method (and code) for modeling packing that uses a more realistic representation of pebble motion gives results nearly identical to the experimental ones (for comparable configuration). This new method will also be incorporated into PEBDAN. Using PEBDAN, this work demonstrated that due to the larger escape probability of neutrons, the Dancoff factors drop several tens of percents along the inner and outer reflector of the core. In the literature the effect of the proximity of reflectors was not accounted for when computing Dancoff factors, whereas Monte Carlo models inherently incorporate all geometric effects, including boundary effects. The new method removes this additional source of discrepancy. It must be noted that the PEBDAN code and the pebble packing fraction modeling codes were not part of the initial task. 


\section{CONTENTS}

ABSTRACT

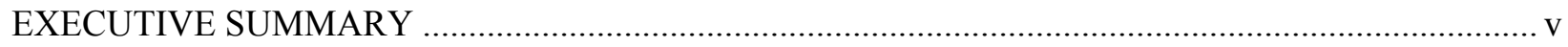

1. INTRODUCTION AND REVIEW OF THE LITERATURE ….............................................. 1

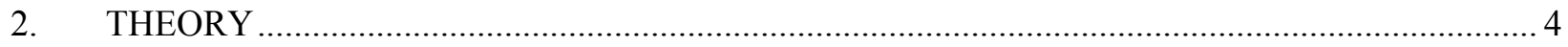

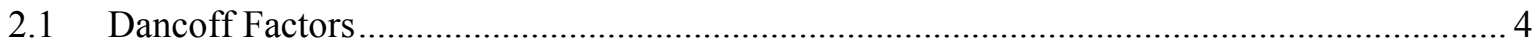

2.2 Infinite-Medium Dancoff Factors According to Bende et al ............................................. 5

2.3 Infinite-Medium Dancoff Factors According to Lane et al................................................. 5

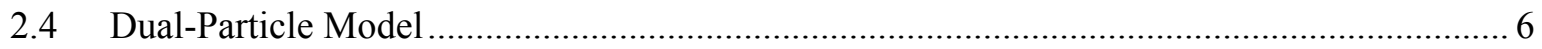

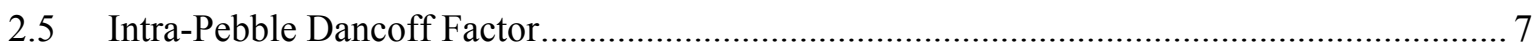

3. RESULTS FOR INFINITE-MEDIUM DANCOFF FACTORS ............................................... 8

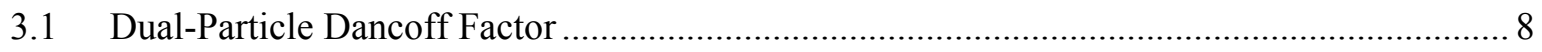

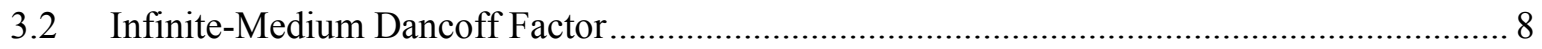

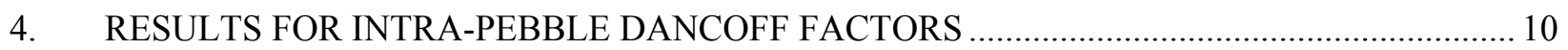

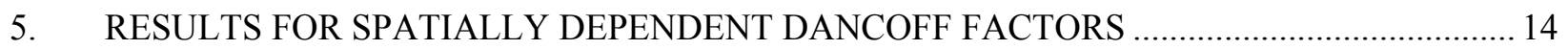

5.1 Generation of Randomly Packed Pebbles ................................................................... 14

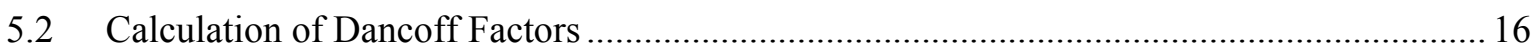

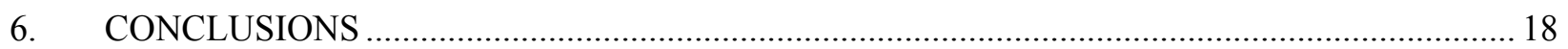

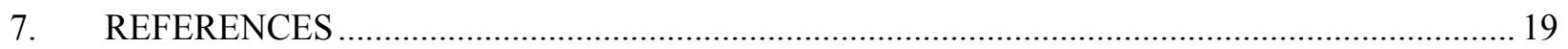

\section{FIGURES}

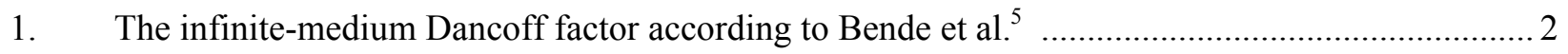

2. The ratio of the infinite-medium Dancoff factor according to Bende et al. ${ }^{5}$ and Lane et al. ${ }^{9} \quad \ldots \ldots . . .3$

3. Geometry for integration in the dual particle model. .............................................................. 7

4. The ratio of the Green's function of the dual-particle model and Lanes'....................................... 8 
5. Percentage differences between infinite-medium Dancoff factors calculated with methods from literature and the dual particle model

6. The ratio of the fuel zone cross section for Lanes' method $^{6}$ and that of Bende. ${ }^{5}$

7. Intra-pebble Dancoff factors as a function of the number of kernels in a standard fuel pebble with a radius of $2.5 \mathrm{~cm}$, with the kernel radius as a parameter.

8. Distribution of the distances traveled by neutrons until their first intersection with another fuel kernel (upper plot), and the cumulative Dancoff factor.

9. Dancoff factor and escape probability as a function of the position in a pebble, with the number of kernels as a parameter $(15,000$ or 30,000 fuel kernels per pebble with fuel zone radius of $2.5 \mathrm{~cm}$ ).

10. The angular distribution (neutrons per unit cosine) escaping from the pebble without interaction with a moderator nuclide and without crossing another fuel kernel.

11. Measured and calculated radial porosity profiles in a cylinder with radius 10.15 times the pebble diameter (0.080 inch).

12. Measured and calculated radial porosity profiles in a cylinder with radius 10.15 times the pebble diameter $(0.080$ inch).

13. Pebble-Pebble Dancoff factor for the NGNP as a function of radial position (top figure) and height (with a reflective boundary at $96 \mathrm{~cm}$ from the bottom). 


\section{Computation of Dancoff Factors for Fuel Elements Incorporating Randomly Packed TRISO Particles}

\section{INTRODUCTION AND REVIEW OF THE LITERATURE}

This report presents the results of a study conducted at the INEEL and partly completed at Delft University of Technology (The Netherlands) to assess the validity for the pebble-bed high-temperature gas-cooled reactor (HTGR) of Dancoff factors, as computed using methods from the literature. As part of this study, a more systematic method for computing Dancoff factors was devised. The motivation for the study derived from observed discrepancies between deterministic predictions (using diffusion theory and cross sections derived using Dancoff factors) on the one hand and MCNP (Monte Carlo neutron-photon) model results on the other. Such studies had been carried out at INEEL, ANL, and Georgia Institute of Technology in the context of the NGNP design and of related projects (NERI), and were reported on previously (some results are referenced herein). A prime source of discrepancy was recognized as the inadequate representation of the random packing of TRISO particles by the various models. This project was therefore proposed to remedy this problem by identifying the best approach possible to modeling the random packing of TRISO particles in the cross section preparation phase. Subsequently, the scope was expanded (at no additional cost) to include modeling the effects of random packing of the pebbles as well as modeling the effect of the proximity of reflectors.

The new method relaxes some of the simplifying assumptions encountered in all previous developments. In particular, the new method rigorously (albeit numerically) accounts for the random packing of the TRISO particles within the pebble. In addition, as stated above, besides the explicit treatment of the random packing of the TRISO particles, the new method accounts for the actual finite geometry of the pebble bed and the proximity of reflectors in determining Dancoff factors. Although the new methods presented here were meant for, and applied to, the pebble bed reactor concept, their conclusions and implications are relevant to any other HTGR fuel shape, including the prismatic block fuel type.

Dancoff factors are used in resonance shielding calculations to adjust the first-flight escape probability of a fuel lump for the probability that a neutron that escapes will enter a neighboring fuel lump without interaction in between. Many programs and subroutines exist that calculate Dancoff factors for regular or irregular lattices, see for example References 1-3. The calculation of Dancoff factors of HTGR fuel types, both pebble-bed and prismatic types, has always been more problematic than that of LWR fuels, because of the six times smaller scatter cross section of the moderator in the resonance region. Consequently, the influence of the double or even triple heterogeneity involved in HTGR fuel types has to be accounted for in the Dancoff factor calculation.

HTGR fuel typically consists of a fuel kernel with a diameter of 350 to $500 \mu \mathrm{m}$, covered by a porous buffer layer and a combination of pyrolytic carbon and SiC layers. The buffer layer accommodates the gaseous fission products that escape from the fuel kernel and protect the outer coating layers from recoiling fission products. The total thickness of all the layers covering the fuel kernel is typically about $200 \mu \mathrm{m}$. The coated particles are embedded in a graphite matrix with either spherical shape for pebble-bed reactors or cylindrical shape for prismatic graphite block types. In pebble-bed reactors, which is the main focus of this report, the spherical fuel zone of a pebble, containing about 10 20 thousand of these coated particles, has a radius of $2.5 \mathrm{~cm}$ and is covered by a graphite layer with a thickness of $0.5 \mathrm{~cm}$. Besides these fuel pebbles, the core may contain moderator pebbles containing graphite but no coated particles, which, from a neutronics point of view, increases the effective thickness of the outer graphite shell of a fuel pebble. 
For pebble-bed reactors, Teuchert accomplished the numerical calculation of Dancoff factors in $1967,{ }^{4}$ while Bende et al. ${ }^{5}$ solved the problem in 1999 analytically (although while still requiring the numerical evaluation of exponential integral functions of the third order). In the latter article, the problem is solved in two steps. First, the Dancoff factor of an infinite number of fuel particles (in an infinite medium) is calculated and corrected for the probability that neutrons may leak from the fuel zone of a pebble to the moderator shell without interaction. This contribution is called the intra-pebble Dancoff factor. Second, the probability that these escaping neutrons may enter a fuel kernel in another pebble, either a neighboring pebble or one further away, is calculated and added to the first term. This latter contribution is called the inter-pebble Dancoff factor. Both contributions can be calculated analytically by using the first-flight escape probability for spheres derived by Case et al., ${ }^{6}$ and the transmission probabilities for spheres derived by Westfall. ${ }^{7}$ For the inter-pebble term, this imposes no approximation as long as no moderator pebbles are admixed with the fuel pebbles. Then, the actual geometry of the physical situation and the geometry used to calculate the escape and transmission probabilities are both spherical.

For the intra-pebble Dancoff factor, however, the underlying assumption that the transmission probability of a large number of fuel kernels randomly distributed in a graphite matrix may be approximated by the moderator transmission probability in a spherical unit cell was never proven, nor was it addressed. For new fuel designs with large fuel kernels at high density, the infinite-medium Dancoff factor based on this assumption may not be accurate. Because the same assumption is implicitly used by resonance shielding codes such as the MICROX-2 $\operatorname{code}^{8}$ used at the INEEL, an assessment of the validity of this formulation, elaborated on in Section 2.2, was needed.

To clarify the potential problem, we show in Figure 1 the infinite-medium Dancoff factor calculated with Bende's method as a function of the fuel kernel radius and the number of fuel kernels in a standard fuel pebble with a radius of $2.5 \mathrm{~cm}$, while in Figure 2 we show the ratio of this Dancoff factor and that of Lane et al. ${ }^{9}$ The latter model is based on a statistical treatment elaborated on in Section 2.3, and is said to be accurate only at high dilution (low particle densities). Surprisingly, the discrepancy between Bende's model and that of Lane is large at high dilution, while at low dilution (high particle densities) there seems to be a nice agreement. The first-mentioned fact is most probably due to the fixed minimum value of the inter-particle spacing in Bende's model, while in a randomly distributed collection of particles, the few particles that are very close to each other contribute significantly to the Dancoff factor. Fortunately, because the Dancoff factor itself is very

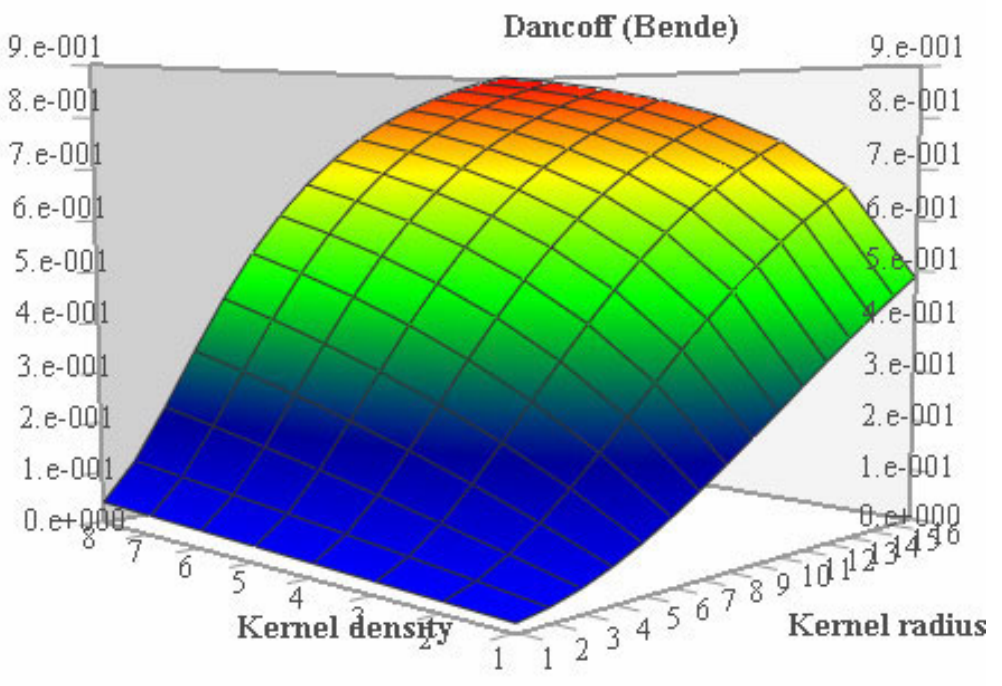

Figure 1. The infinite-medium Dancoff factor according to Bende et al. ${ }^{5}$ The kernel radius is given in units of $25 \mu \mathrm{m}$ (maximum is $400 \mu \mathrm{m}$ ), the density in units of 5,000 , in a standard fuel pebble (maximum is 40,000 ). low at high dilution, this discrepancy is of low significance. The second-mentioned fact, the good agreement at high particle density, may be more serious, as we are not aware of any seriously limiting assumption in Bende's method in this region. 
In Section 2, some theory about Dancoff factors is reviewed:

Section 2.1 discusses the application of Dancoff factors in resonance shielding calculations, Section 2.2 the calculation of infinite-medium Dancoff factors according to Bende's model, and Section 2.3 the statistical model of Lane. In Section 2.4, we discuss some methods to test the applicability of the two previously mentioned models for lattices with a high packing fraction. Section 2.5, finally, describes the extension of infinite-medium Dancoff factors to intra-pebble Dancoff factors. Results about this issue are given in Section 3.

The second issue addressed in this report is the influence of the particle distribution in the fuel pebble

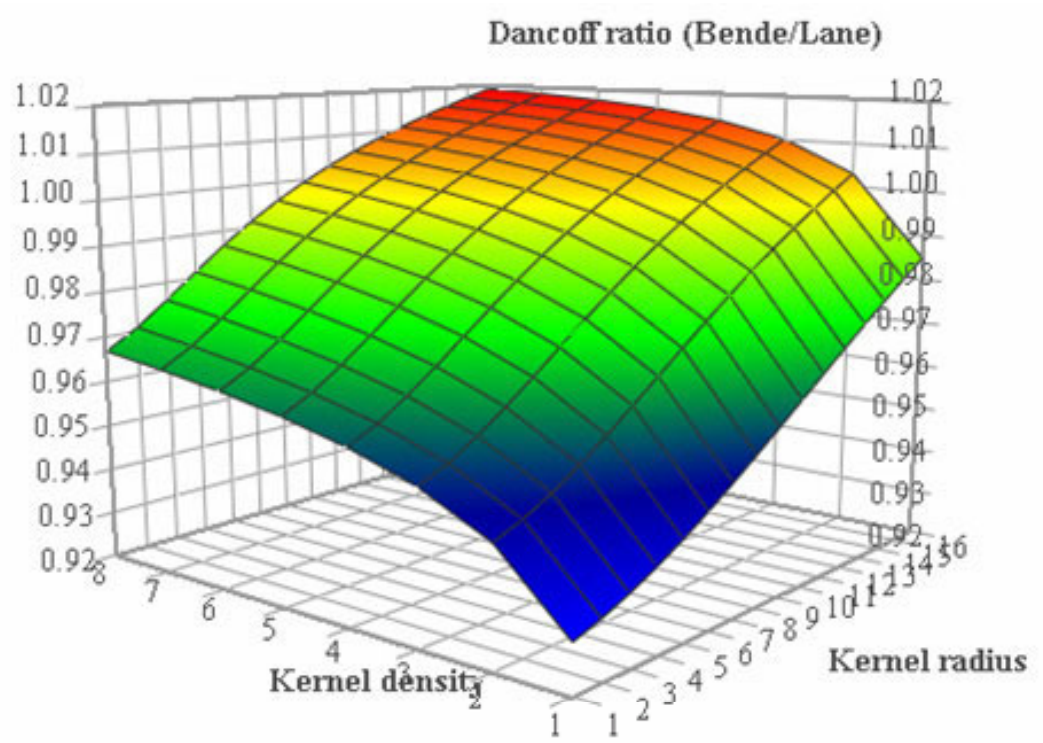

Figure 2. The ratio of the infinite-medium Dancoff factor according to Bende et al. ${ }^{5}$ and Lane et al. ${ }^{9}$ The kernel radius is given in units of $25 \mu \mathrm{m}$ (maximum is $400 \mu \mathrm{m}$ ), the density in units of 5,000, in a standard fuel pebble (maximum is 40,000). on the Dancoff factor. In a recent paper, ${ }^{10} \mathrm{Kim}$ et al. found that the eigenvalues of the Next Generation Nuclear Plant (NGNP) under study at INEEL differ significantly for a random distribution of fuel kernels and for a regular lattice, with the latter values always being lower. They found that this discrepancy is due to an overestimation of the neutron capture rate in the main resonance absorbers by about $1 \%$. To investigate and verify this effect, a Monte-Carlo program was written that calculates the intra-pebble Dancoff factor for random and cubic packing of particles in the fuel pebble. Besides the pebble-average Dancoff factors, this program also calculates the radial dependence of Dancoff factors, and the escape probability as well as the angular distribution of the outgoing current. Results are given in Section 4.

The third issue addressed in this report concerns the calculation of space-dependent Dancoff factors for pebble-bed reactors. Because of enhanced neutron leakage, the inter-pebble Dancoff factor will be lower at the boundary of a reactor core. For water-moderated reactors, this effect is quite small because of the small diffusion length, but for graphite-moderated reactors, the effect might be more significant. A Monte-Carlo program was written that calculates the different contributions to the Dancoff factor as a function of radius and height in a cylindrical reactor core (although the latter is not a limitation of the raytracing method, and other geometries could be easily programmed as well). Results are given in Section 5. The report's conclusions are given in Section 6 


\section{THEORY}

\subsection{Dancoff Factors}

As mentioned, the application of Dancoff factors arises in the calculation of group-wise resonance shielded cross sections. Most resonance shielding codes solve the two-region slowing-down equation:

$\Sigma_{t}^{F}(E) \Phi_{F}(E)=\left[1-P_{F M}(E)\right] \int_{E}^{E / \alpha_{F}} d E^{\prime} \frac{\Sigma_{s}^{F}\left(E^{\prime}\right) \Phi_{F}\left(E^{\prime}\right)}{\left(1-\alpha_{F}\right) E^{\prime}}+\frac{P_{F M}(E) \Sigma_{t}^{F}\left(E^{\prime}\right)}{E}$

where the symbols have their usual meaning (see for example Chapter 10 in Reference 11). The probability $\mathrm{P}_{\mathrm{FM}}$ accounts for the chance that a neutron that escapes from a fuel lump will have its next interaction in the moderator. The equation above is solved for the spatial average of the neutron flux in the fuel lump. In deriving this equation, several assumptions were made: the neutron slowing down source in the fuel lump and in the moderator should be spatially flat (flat source approximation), such that the reciprocity theorem ${ }^{12}$ may be applied, the energy loss per collision with a moderator nuclide should be much larger than the width of the resonance (narrow resonance approximation for moderator nuclides), and no absorption should take place in the moderator.

For isolated lumps, the first-flight escape probability, which were derived analytically for several geometries by Case et al., ${ }^{6}$ can be substituted for $P_{F M}$ without large error. However, when resonance neutrons that escape from a fuel lump can enter another lump, a correction to the first-flight escape probability has to be made, as explained in Reference12. A neutron that escapes from a fuel lump will have a probability $P_{M}^{0}$ to have its first subsequent interaction with a moderator nuclide surrounding that lump, a probability $\left(1-P_{M}^{0}\right)\left(1-P_{F}^{0}\right) P_{M}^{1}$ to that first interaction with a moderator nuclide after traversing one other fuel lump, a probability $\left(1-P_{M}^{0}\right)\left(1-P_{F}^{0}\right)\left(1-P_{M}^{1}\right)\left(1-P_{F}^{1}\right) P_{M}^{2}$ to have that first interaction with a moderator nuclide after traversing a second fuel lump, etc. The probability that the first interaction be with a moderator atom after any number of transits through fuel lumps is the sum of all these probabilities, which is the sum of an infinite series. Assuming $P_{M}^{i}=P_{M}^{0}$ and $P_{F}^{i}=P_{F}^{0}$ for all $i>0$, this sum of probabilities converges, and the total probability for a neutron to escape from a fuel lump becomes:

$P_{F M}=P_{e s c} \frac{P_{M}^{0}}{1-\left(1-P_{M}^{0}\right)\left(1-P_{F}^{0}\right)}=P_{e s c} \frac{1-C}{1-C\left(1-P_{F}^{0}\right)}$.

Here $P_{\text {esc }}$ is the first-flight escape probability, and $C=1-P_{M}^{0}$ is the Dancoff factor, which is equal to the probability for a neutron that escapes from a fuel lump to enter another lump without interaction with a moderator nuclide in between. Note that the "gray" effect, which accounts for the probability that a neutron may traverse one or more fuel lumps without interaction, is already accounted for in the Dancoff factor itself. To calculate the probabilities $P_{M}^{0}$ and $P_{F}^{0}$, one usually assumes that all neutrons escaping from a fuel rod and all neutrons entering a moderator zone have an isotropic angular distribution. This means that in calculating the transmission probabilities, each surface can be considered as a white boundary source, which practically translates into the assumption of a cosine angular current through that surface. The validity of this assumption is discussed in Section 4. 


\subsection{Infinite-Medium Dancoff Factors According to Bende et al.}

In the method of Bende and his co-workers, ${ }^{5}$ the transmission probabilities are calculated in a spherical unit cell containing a fuel kernel and a moderator zone with thickness that preserves all moderator nuclides in the fuel zone of a pebble. The moderator zone thus includes the carbon and silicon atoms from the coatings that cover each fuel kernel, but excludes the carbon atoms from the 0.5 -cm-thick outer shell of the fuel pebble. The infinite-medium Dancoff factor then becomes the product of the probability of a neutron emitted from a fuel kernel to reach the outer boundary of the spherical unit cell $\left(t_{i o}\right)$, the probability to cross the moderator zone of an arbitrary number of $n$ adjacent unit cells without entering a fuel kernel $\left(t_{o o}^{n}\right)$, and the probability of a neutron emitted from the outer boundary of the moderator zone to reach another fuel kernel $\left(t_{o i}\right)$. In accordance with the theory described in the previous section, these transmission probabilities, reported for spherical geometry by Westfall, ${ }^{7}$ should be calculated assuming a white boundary. In equation form, the Dancoff factor becomes:

$$
C_{\infty}^{\text {Bende }}=\sum_{n=0}^{\infty} t_{i o} t_{o o}^{n} t_{o i}=\frac{t_{i o} t_{o i}}{1-t_{o o}}
$$

Note that because of the way the unit cell is defined, in this formalism a neutron always travels through the moderator zone of the coated particle that includes the average amount of moderator nuclides corresponding to the graphite located between the coated particles. The minimum distance a neutron has to travel to another kernel is therefore always larger then the distance between two coated particles adjacent to each other, which may give a biased value for the Dancoff factor at low dilution.

\subsection{Infinite-Medium Dancoff Factors According to Lane et al.}

In 1962, Lane et al. ${ }^{9}$ derived the infinite-medium Dancoff factor for randomly distributed particles. If $m$ is the particle density, the macroscopic cross section for crossing a fuel kernel equals $\Sigma_{t}^{F}=m \pi r_{1}^{2}$, where $r_{1}$ is the geometrical radius of the kernel. The probability that the volume element $d V$ contains one or more particles equals $\sum_{n=1}^{\infty} \frac{(m d V)^{n}}{n !} \exp (-m d V) \approx m d V$. The probability that a ray of length $r$ does not cross a fuel kernel equals $\exp \left(-\Sigma_{t}^{F} r\right)$, while the probability that a neutron along the same ray does not interact with a moderator nuclide equals $\exp \left(-\Sigma_{t}^{M} r\right)$. Using these probabilities, the infinite-medium Dancoff factor becomes:

$$
C_{\infty}^{\text {Lane1 }}=\int_{0}^{\infty} \exp \left[-\left(\Sigma_{t}^{F}+\Sigma_{t}^{M}\right) r\right] m \pi r_{1}^{2} d r=\frac{\Sigma_{t}^{F}}{\Sigma_{t}^{M}+\Sigma_{t}^{F}}=\frac{1}{1+\Sigma_{t}^{M} \bar{l}}
$$

with $\bar{l}=4\left(V_{M}+V_{F}\right) / S_{F}$ is some kind of average chord length. ${ }^{13}$ If all the particles contain a coating with thickness $c$, the lower limit for the integral should be $2 c$ instead of 0 , and the Dancoff factor changes accordingly ${ }^{9}$ to

$$
C_{\infty}^{\text {Lane } 2}=\frac{\Sigma_{t}^{F}}{\sum_{t}^{M}+\Sigma_{t}^{F}} \exp \left[-2 c\left(\Sigma_{t}^{M}+\Sigma_{t}^{F}\right)\right]
$$


However, for many cases considered in this report, this modification gives worse results than the previous formula.

In conjunction with this model, a Green's function can be defined that gives the contribution to the Dancoff factor of two particles a distance $r$ apart with only moderator in between. This Green's function reads $G^{\text {Lane }}(r)=\exp \left(-\Sigma_{t}^{M} r\right) r_{1}^{2} / 4 r^{2}$ and accounts for the fraction of the isotropic source emitting neutrons in solid angle $\pi r_{1}^{2} / r^{2}$. The infinite-medium Dancoff factor according to Lane's model can then be restated as

$$
C_{\infty}^{\text {lane1 }}=\int_{0}^{\infty} G^{\text {Lane }}(r) \exp \left(-\Sigma_{t}^{F} r\right) 4 \pi r^{2} d r
$$

which will be the form used for numerical comparison with the dual-particle model introduced in the next section.

Note that a slight modification can be made to Lane's model by adjusting the moderator cross section with the volume fraction occupied by the fuel kernels according to $\Sigma_{t}^{M^{*}}=\Sigma_{t}^{M}\left(1-m \frac{4 \pi}{3} r_{1}^{3}\right)$. In this case, one gets a similar expression for the Dancoff factor,

$$
C_{\infty}^{\text {Janssen }}=\int_{0}^{\infty} \exp \left[-\left(\Sigma_{t}^{F}+\Sigma_{t}^{M^{*}}\right) r\right] m \pi r_{1}^{2} d r=\frac{\Sigma_{t}^{F}}{\Sigma_{t}^{M^{*}}+\Sigma_{t}^{F}}=\frac{1}{1+\Sigma_{t}^{M} \bar{l}^{*}}
$$

but with a slightly different chord length given by $\bar{l}^{*}=4 V_{M} / S_{F}$. Janssen originally derived this approximation in a memorandum that has not been widely distributed. ${ }^{14}$

\subsection{Dual-Particle Model}

In the previous section, the kernel size was assumed to be infinitely small, which is likely to give deviations for large kernels. Therefore, we derived a Green's function that describes the probability for a neutron escaping from the surface of one fuel kernel to reach another fuel kernel with its center a distance $d$ away without interaction with moderator nuclei in between. This Green's function, which can be considered as a dual particle Dancoff factor, is given by

$$
G^{\text {Dual }}(d)=\frac{\int_{A_{2}\left(\mu_{2}<0\right)} \int_{A_{1}\left(\mu_{1}>0\right)} \exp \left(-\Sigma_{t}^{M} s\right) \mu_{1} d A_{1}\left|\mu_{2}\right| d A_{2}}{4 \pi^{2} r_{1}^{2} s^{2}} .
$$

The areas and angles are shown in Figure 3. The integration over area $A_{l}$ of the emitting kernel is only to be performed for outgoing directions $\left(\mu_{1}>0\right)$, while the integration over area $A_{2}$ of the receiving kernel is only for inward directions $\left(\mu_{2}<0\right)$. Because the integration over the angle $\phi_{1}$ can be carried out analytically, the surface element $d A_{1}$ reduces to $d A_{1}=2 \pi r_{1}^{2} \sin \theta_{1} d \theta_{1}$; in contrast $d A_{2}$ remains $d A_{2}=r_{1}^{2} \sin \theta_{2} d \theta_{2} d \phi_{2}$. The distance $s$ is measured from $d A_{1}$ to $d A_{2}$, and approaches $d$, the distance 
between the kernel centers, with little relative error when the particles are widely spaced. This Green's function accounts for the geometrical size of the particles, and can directly be compared with that of Lane's model. Integration from zero to infinity, weighted with the probability that there are no fuel kernels in between, gives the infinite-medium Dancoff factor. This weighting process needs some care, however. The ray from the surface of one kernel to another, could intersect a third kernel only if the distance between the coatings of the emitting and receiving kernel is larger than $2 \sqrt{r_{2}^{2}-r_{1}^{2}}$, with $r_{1}$ the fuel kernel radius, and $r_{2}$ the outer radius of the coating. For distances less, there can only be moderator in between.

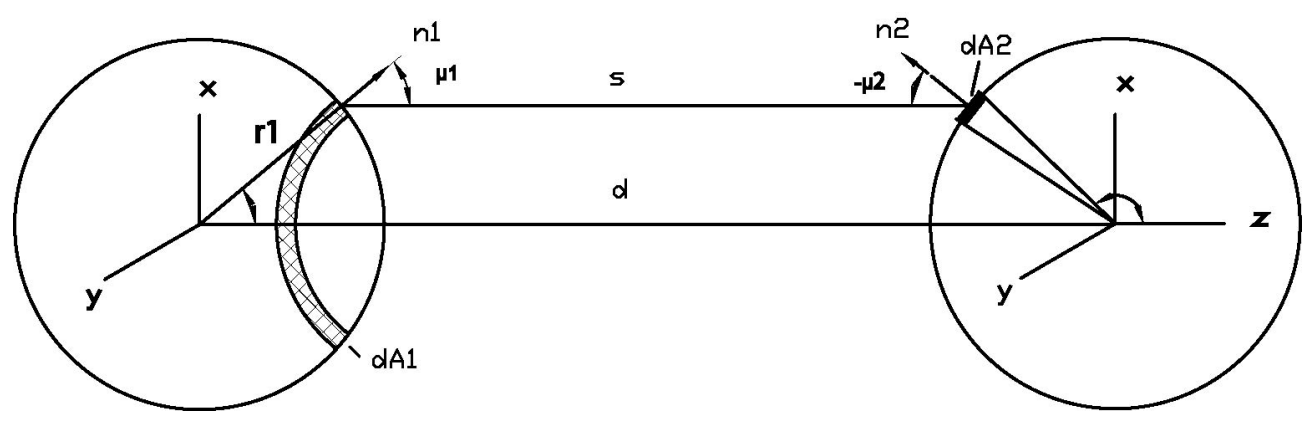

Figure 3. Geometry for integration in the dual-particle model.

\subsection{Intra-Pebble Dancoff Factor}

The intra-pebble Dancoff factor is obtained by volume averaging the Green's function over the entire volume of the fuel zone of a pebble. Because of symmetry reasons, this volume integral reduces to an integral over the cross sectional area of the pebble. ${ }^{5}$ The result can be expressed in terms of the firstflight escape probability $P_{e s c}$ originally derived by Case et al.: ${ }^{6}$

$$
C_{\text {Intra }}=C_{\infty}^{f k}\left[1-P_{e s c}\left(\Sigma^{*} R_{1}\right)\right] \text {. }
$$

The argument of the first-flight escape probability function is the product of the cross section of the fuel zone (the probability per unit length to interact with a moderator nuclide, or to intersect another fuel kernel), and the radius of the fuel zone of the pebble. For Lane's method, this cross section simply becomes the sum of the moderator cross section and the fuel cross section given by $\Sigma_{t}^{F}=m \pi r_{1}^{2}$, with $m$ the particle density, and $r_{1}$ the radius of the fuel kernel. For Bende's method, the result is more complicated (see Reference 5). 


\section{RESULTS FOR INFINITE-MEDIUM DANCOFF FACTORS}

\subsection{Dual-Particle Dancoff Factor}

In Figure 4 we compare the Green's function of the dual-particle model with that of Lane's for fuel kernel radii varying from 25 to 400 $\mu \mathrm{m}$, and for particle densities up to 40,000 per standard fuel pebble. Clearly, for large radii and large densities (low dilution), the discrepancy between the two functions reaches about $10 \%$. However, one must realize that the packing fraction in this region is about $55 \%$, which is almost equal to the maximum achievable for randomly packed beds (62\%). Such high packing fractions are not likely in pebble-bed HTR fuel designs for which packing of the order of $10 \%$ is expected. In contrast, the packing fraction might reach intermediate to high values $(30 \%)$ in prismatic fuel designs. Nevertheless, based on these results, one may expect (small) differences in Dancoff factors as well.

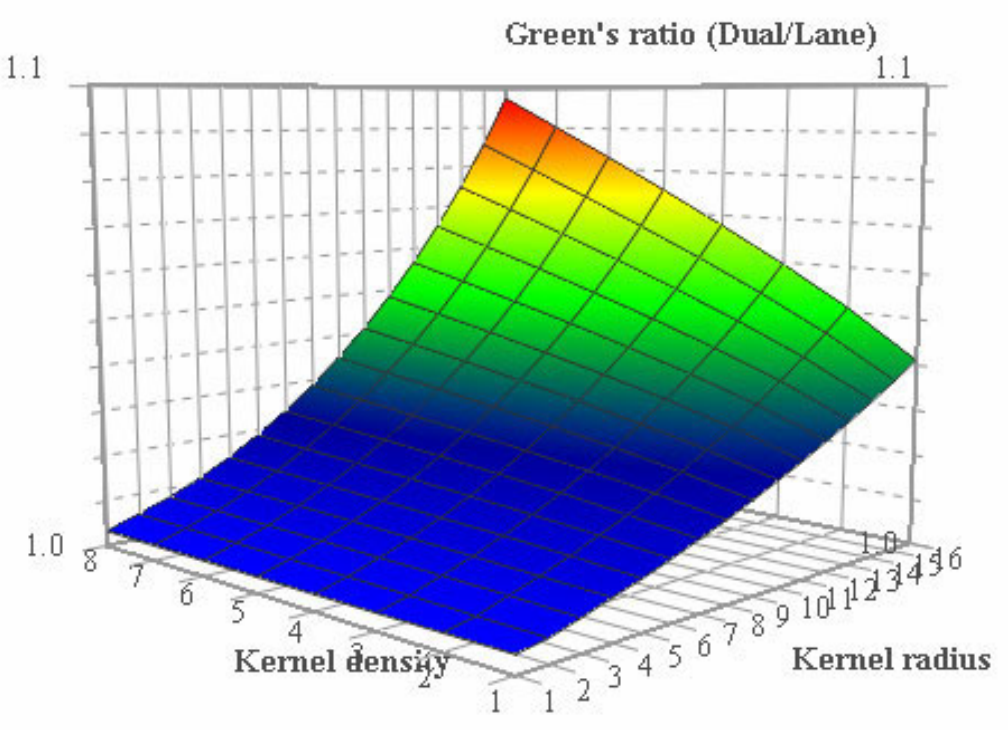

Figure 4. The ratio of the Green's function of the dualparticle model and Lanes'. The kernel radius is given in units of $25 \mu \mathrm{m}$ (maximum is $400 \mu \mathrm{m}$ ), the kernel density in units of 5,000 in a standard fuel pebble (maximum is 40,000). For large kernels at high density, Lane's model ${ }^{9}$ underestimates the probability for a neutron to reach a neighboring fuel kernel by about $10 \%$.

\subsection{Infinite-Medium Dancoff Factor}

Integrating the Green's function of the dual-particle model over distance $d$ ranging from $2 r_{1}$ to $\infty$, or that of Lane's model from 0 to $\infty$, gives the infinite-medium Dancoff factor. Of course, if the integration is performed accurately enough, the latter method gives results equal to the analytical ones given in Equation (4), and care is taken that this is indeed the case. In Figure 5, we compare the four models from the literature given in Equations (3), (4), (5), and (7) with ours. There are few remarkable results; the simplest formula to calculate infinite-medium Dancoff factors, Lane's model given in Equation (4) (Lane1), gives remarkably good results, while the modification for particles with fixed coating [Lane2, Equation (5)], improves slightly the results at high dilution, but worsens them considerably at low dilution. The reason for this is that the Green's function according to Lane's model gives smaller values than that of the dual particle model (see Figure 4), which is compensated in the Lane1 model by an extra contribution in the integral [Equation (4)] starting at zero instead of twice the thickness of the coating layers $(2 c)$. 

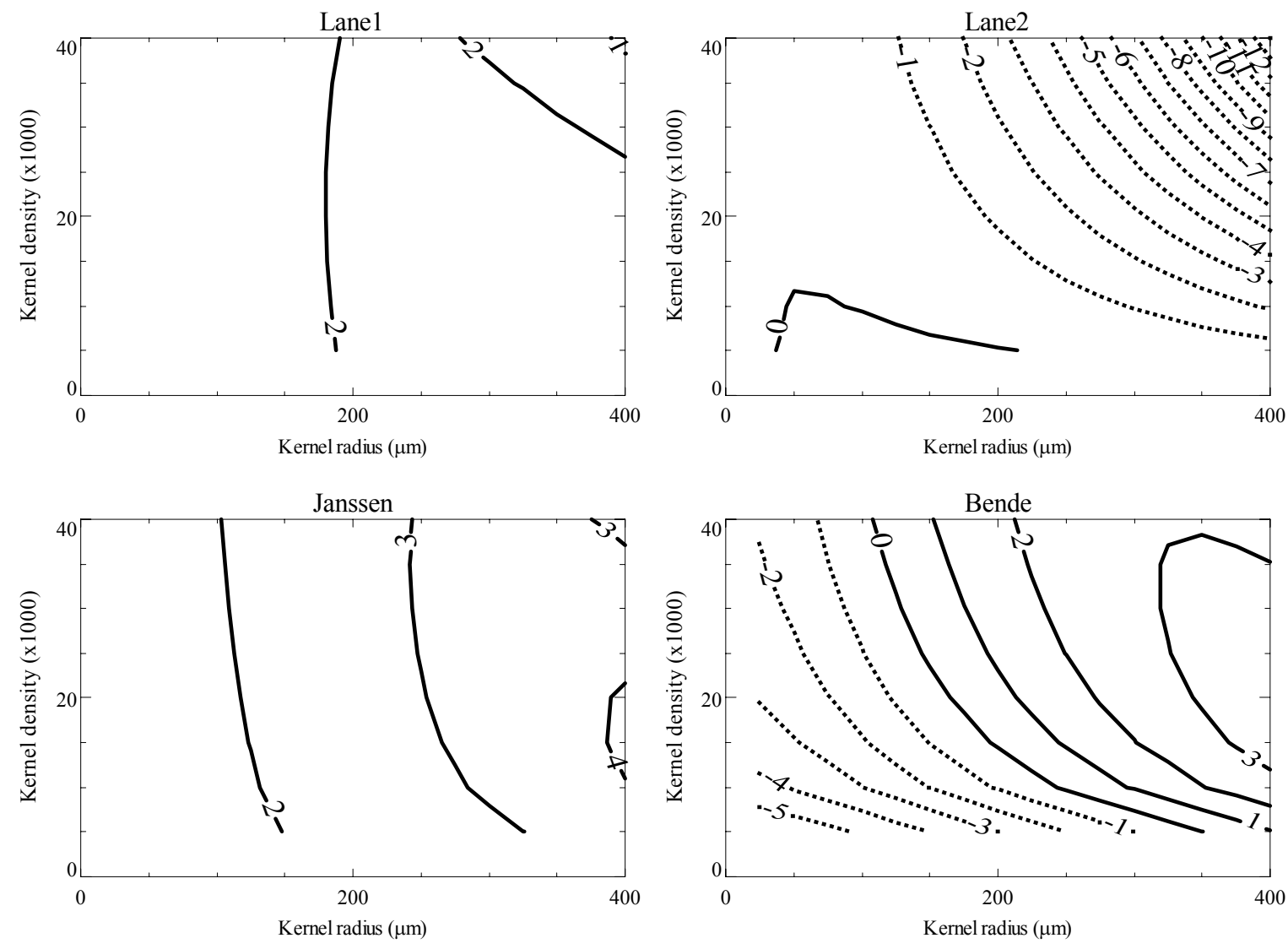

Figure 5. Percentage differences between infinite-medium Dancoff factors calculated with methods from literature and the dual particle model. The coating thickness is $200 \mu \mathrm{m}$. Dotted lines show negative differences, solid lines positive.

Janssen's rational approximation gives reasonably good results as well, while Bende's model shows a tendency to underestimate Dancoff factors at high dilution by 4 to $5 \%$. This latter fact is also recognized in Reference 5, which gives confidence in our model. At high density, the discrepancy is no more than $2 \%$, which is quite acceptable. For all practical cases considered (i.e., cases in the design range of the NGNP pebble bed fuel), Bende's model agrees with ours within $1 \%$.

The results in the previous two sections were calculated for a coating with thickness of $200 \mu \mathrm{m}$, which is a good approximation for coatings considered nowadays, which range from $160 \mu \mathrm{m}$ for the HTTR $^{16}$ to $216 \mu \mathrm{m}$ for the HTR-10. ${ }^{17}$ To check the dependency on the coating, calculations were repeated for thickness of 150 and $250 \mu \mathrm{m}$, but differences turned out to be much smaller than the percentage differences shown in Figure 5. 


\section{RESULTS FOR INTRA-PEBBLE DANCOFF FACTORS}

To investigate the effect of the kernel packing on the Dancoff factor, a Monte Carlo program, called INTRAPEB, was written that calculates the Dancoff factor, the distribution of distances traveled by neutrons until their first intersection with another fuel kernel, the escape probability, and the angular distribution of the escaping neutrons. In this program, the kernels are positioned either in a cubic lattice, or in a random lattice. The latter is done for each particle by drawing a random position, and checking whether there is no overlap with any other particle (kernel with surrounding coatings). After positioning all particles, a kernel is selected randomly, as well as the position and angular distribution of the emerging neutron. The distances between this escape position and all other particles traversed by the ray are calculated to obtain the first intersection of the ray with a fuel kernel. This process is repeated many times (typically between 100,000 and 1,000,000) to obtain statistically small errors for the Dancoff factor. If there is no fuel kernel intersection, the neutron, corrected for the probability that it might have had a collision with a moderator nucleus, contributes to the escape probability. In this way, the the intra-pebble Dancoff factors are calculated with a relative standard deviation $<<1 \%$.

Besides the Monte-Carlo-based Dancoff factors, INTRAPEB also calculates the intra-pebble Dancoff factor based on the methods of Bende and Lane. For both methods, this factor depends on the infinite-medium Dancoff factor shown in Figure 2, and on the cross section of the fuel zone $\left[\Sigma^{*}\right.$ in Equation (9)]. The ratio of the latter parameters is shown in Figure 6. Clearly, at high packing fraction, the cross sections are not the same, which is due to the fact that in Lane's method, the fuel kernels are assumed to be infinitely small, which is not a valid assumption anymore in the low dilution region (i.e., for high packing cases). Fortunately, because $\Sigma^{*}$ becomes very large in this region (between $3-4 \mathrm{~cm}^{-1}$ ), due to the large packing fraction, the escape probability becomes very small, and the final result is not very sensitive to the value of $\Sigma^{*}$. On the other hand, the infinite-medium Dancoff factors are pretty much the same in this region (see Figure 2).

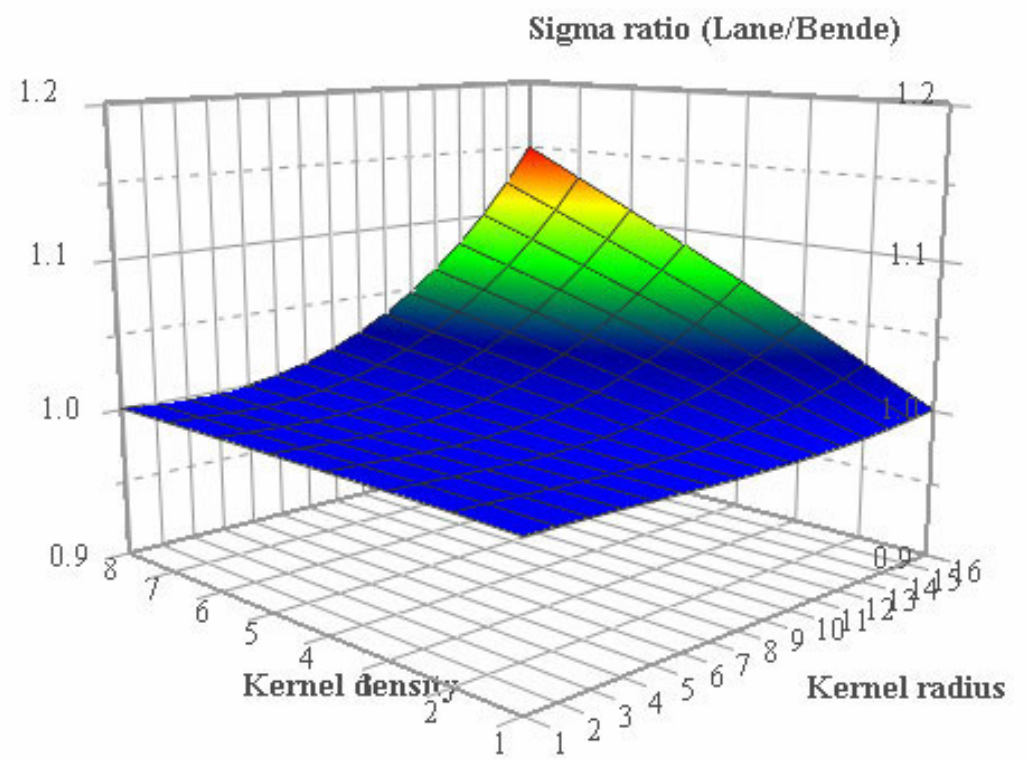

Figure 6. The ratio of the fuel zone cross section for Lanes' method ${ }^{6}$ and that of Bende. ${ }^{5}$ The kernel radius is given in units of $25 \mu \mathrm{m}$ (maximum is $400 \mu \mathrm{m}$ ), the density in units of 5,000 in a standard fuel pebble (maximum is 40,000 ).

In Figure 7, we show the intra-pebble Dancoff factor as a function of the kernel density in a standard pebble with a fuel zone radius of $2.5 \mathrm{~cm}$ for kernel radii of 100 and $250 \mu \mathrm{m}$. Clearly, the cubic lattice gives Dancoff factors significantly higher than the stochastic lattice. This is in agreement with the observations made in Reference 10, where it was found that the eigenvalue for the Next Generation Nuclear Plant is consistently lower for a regular packing of fuel particles due to increased resonance absorption. 


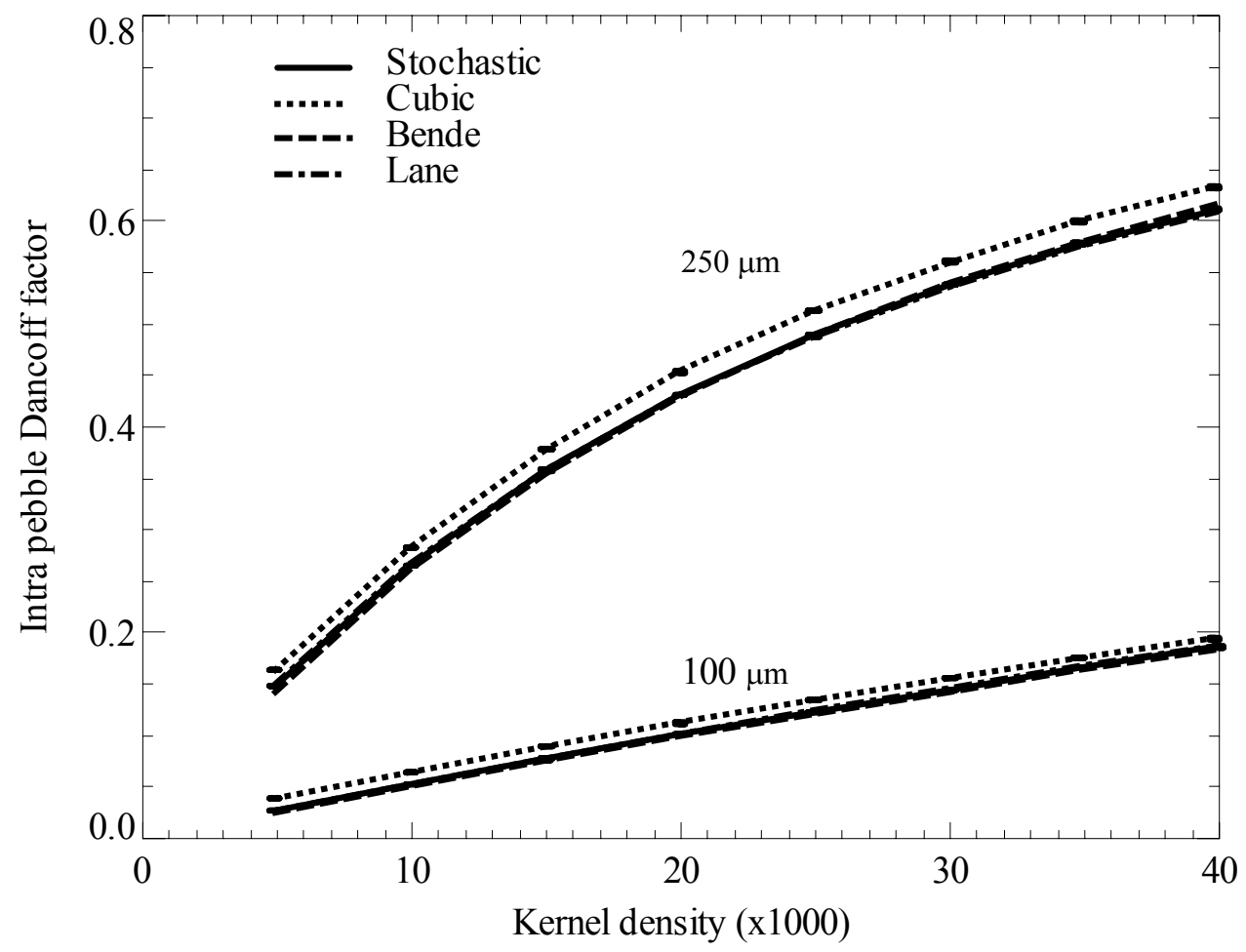

Figure 7. Intra-pebble Dancoff factors as a function of the number of kernels in a standard fuel pebble with a radius of $2.5 \mathrm{~cm}$, with the kernel radius as a parameter. Clearly, the Dancoff factors for a cubic lattice are larger than for the other methods. The standard deviations in the results for cubic and random lattices are much less then $1 \%$.
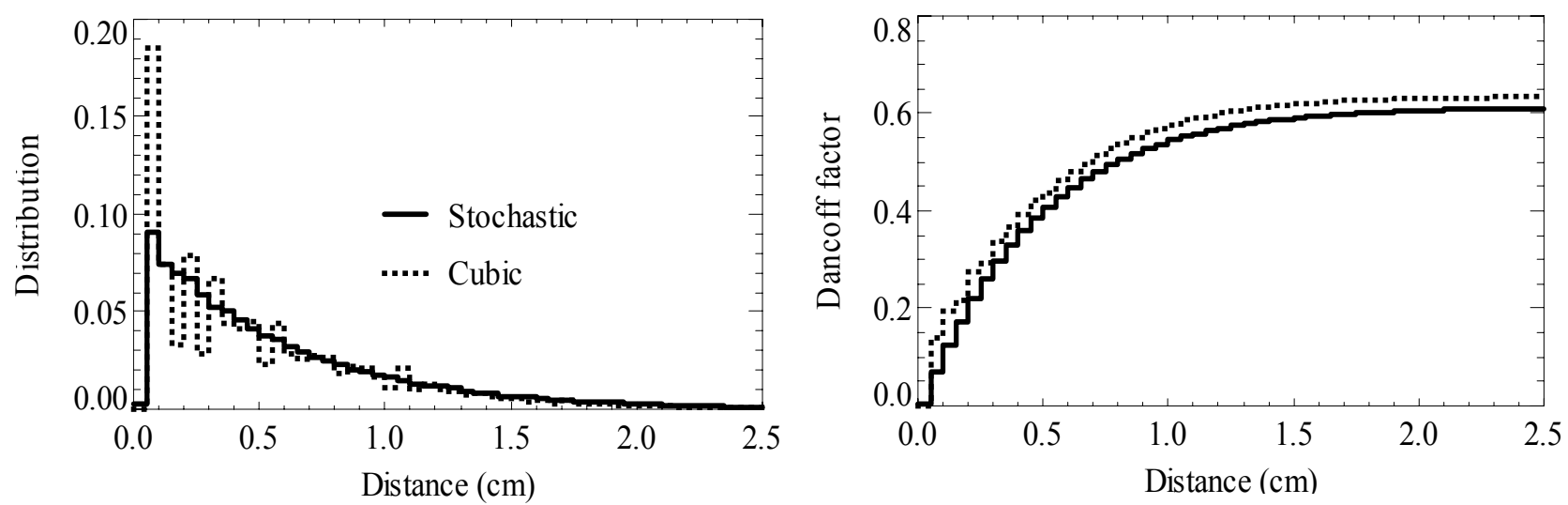

Figure 8. Distribution of the distances traveled by neutrons until their first intersection with another fuel kernel (upper plot), and the cumulative Dancoff factor. In a cubic lattice, neutrons have a large probability to hit one of the nearest neighbors, which results in a relatively large contribution to the Dancoff factor. This is for quite an extreme case, with a particle density of 40,000 per standard fuel pebble, and a fuel kernel radius of $250 \mu \mathrm{m}$.

However, the results found here are in contradiction with observations made in Reference 5, where it was found that for kernels with a radius of $100 \mu \mathrm{m}$, Monte Carlo calculations with MCNP gave 
consistently lower Dancoff factors. This was attributed to channel ray effects that would enhance neutron leakage. Although these channel ray effects might exist for scattered neutrons, they could not be confirmed for the unscattered neutrons that make up the contribution to the Dancoff factor. In Figure 8 we show that in a cubic lattice, relatively many neutrons, much more than in a stochastic lattice, intersect the nearest kernels, which results in a relatively high Dancoff factor. Furthermore, for kernels with a radius of $250 \mu \mathrm{m}$, the escape probability in a cubic lattice is lower than in a random lattice for all particle densities considered here. The differences increase with particle density, and range from $2 \%$ at 5,000 particles per pebble, to $6 \%$ at 40,000 particles).

In Figure 9, the intra-pebble Dancoff factor is shown for a single pebble as a function of the radial position in the fuel zone of the pebble, together with the escape probability. Clearly, both parameters depend strongly on the position in the pebble. For the 15,000 and 30,000 particle cases, the average values of the Dancoff factor reach 0.358 and 0.540, respectively, which agree within $1 \%$ with the methods of Bende, Lane, and Janssen. The neutrons that escape from the pebble contribute partly to the interpebble Dancoff factor, which means that the total Dancoff factor (the sum of the intra- and inter-pebble contributions) shows a less pronounced dependency on position (see Figures 9 and 10 in Reference 5).

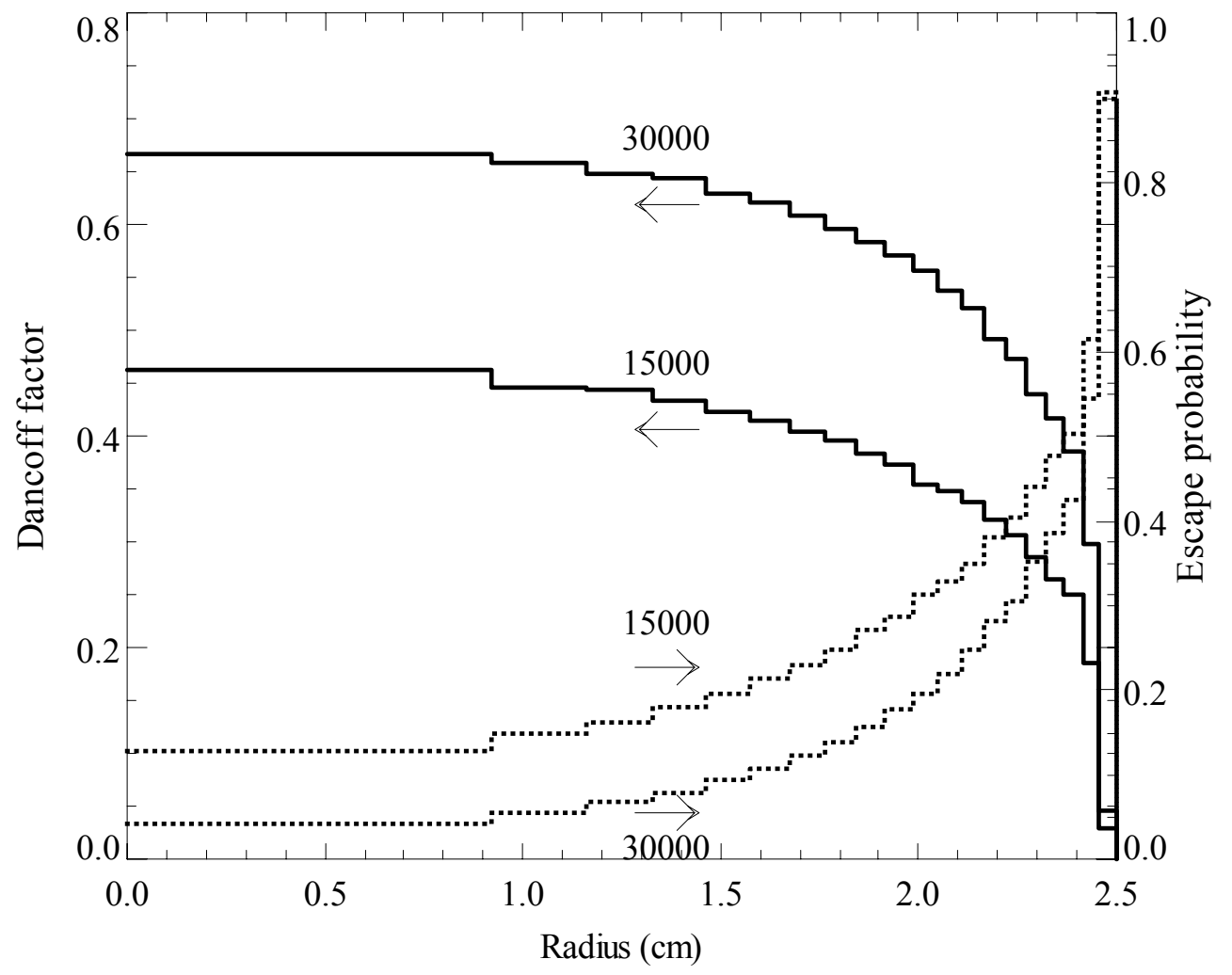

Figure 9. Dancoff factor and escape probability as a function of the position in a pebble, with the number of kernels as a parameter $(15,000$ or 30,000 fuel kernels per pebble with fuel zone radius of $2.5 \mathrm{~cm})$. The fuel kernel radius equals $250 \mu \mathrm{m}$. Clearly, the Dancoff factor decreases toward the outer boundary of the pebble, whereas the escape probability increases. 
In Figure 10, we show the angular distribution of neutrons escaping from the pebble without any interaction and without crossing another fuel kernel. For the boundary between the fuel zone and the moderator layer, the cosine distribution seems a reasonable assumption, but the angular distribution at the outer boundary of the moderator layer is much more forwardly peaked. This means that the white boundary condition assumed in the calculation of transmission probabilities (see Sections 2.1 and 2.2) is not fully valid.

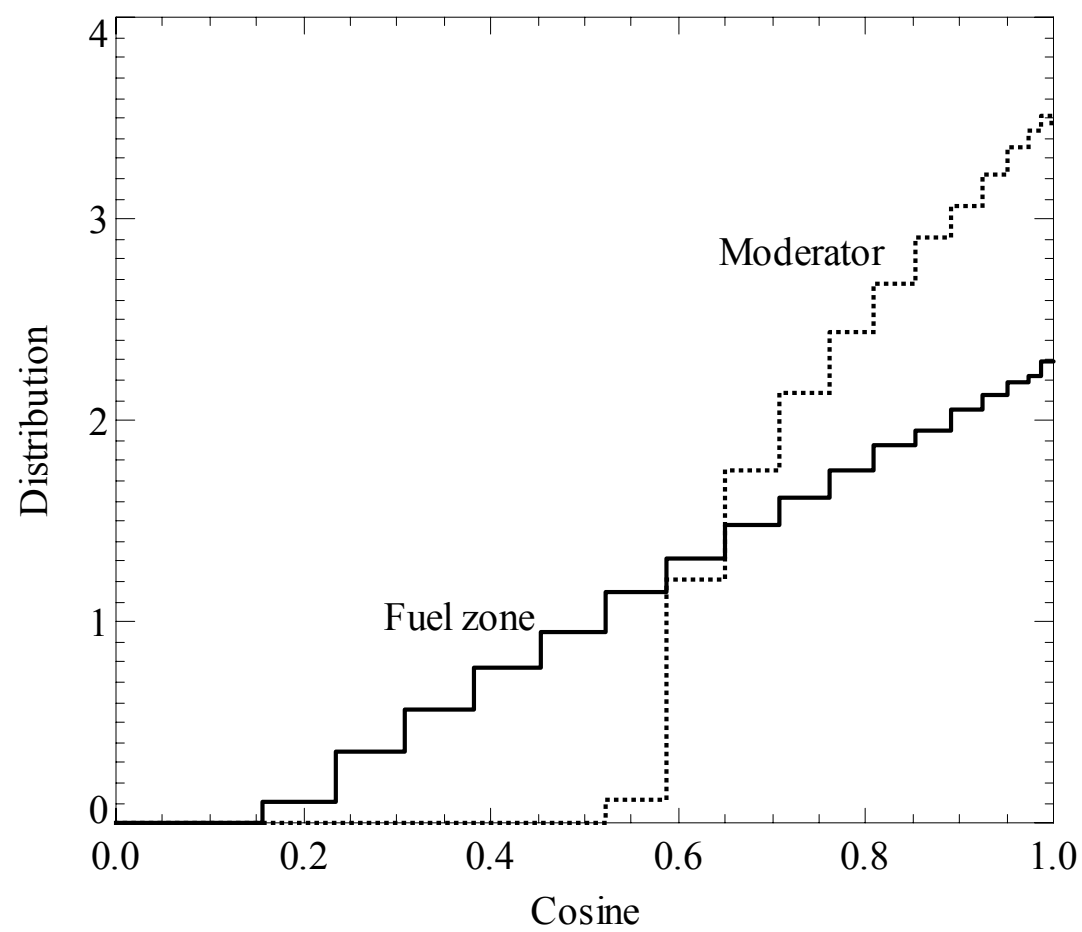

Figure 10. The angular distribution (neutrons per unit cosine) escaping from the pebble without interaction with a moderator nuclide and without crossing another fuel kernel. Clearly, the distribution of neutrons escaping the fuel zone is reasonably cosine distributed, but that of neutrons escaping the moderator zone is much more forwardly peaked. This figure is for a standard pebble (radii 2.5 and $3.0 \mathrm{~cm}$ for the fuel zone and moderator) containing 15,000 kernels, with a radius of $250 \mu \mathrm{m}$, but the results for 30,000 kernels are very similar. 


\section{RESULTS FOR SPATIALLY DEPENDENT DANCOFF FACTORS}

The results presented in the previous section clearly indicate that the intra-pebble Dancoff factor depends on the radial position in the fuel zone of a pebble. In practice, it will be very difficult to account for this effect, as it would require dividing the pebbles in the core in radial zones and performing cross section treatment for each zone. However, a similar effect can be expected for the inter-pebble Dancoff factor, as neutrons originating near the boundary of the reactor core have a larger escape probability than other neutrons. Dividing the core into regions with different cross section sets is common practice, and one could provide each region with its own Dancoff factor. This would especially be useful for resonance shielding codes that account for the intra-pebble Dancoff factor (or equivalent treatment) themselves and that only need the inter-pebble Dancoff factors or the pebble-pebble Dancoff factors to be given in the input (the pebble-pebble Dancoff factor is defined as the probability that a neutron escaping from the fuel zone of a pebble enters another fuel zone). This is the case for the MICROX-2 code ${ }^{8}$ in use at INEEL.

A program called PEBDAN was written that calculates the spatially dependent Dancoff factors in a cylindrical core with outer and inner reflectors. The problem tackled in this code can be divided into two large portions: (1) calculation of the pebble coordinates randomly stacked in the core volume and (2) the computation of the Dancoff factors proper.

\subsection{Generation of Randomly Packed Pebbles}

The literature on the first problem can be divided into two approaches. One approach is to use rigorous algorithms that simulate pebble flow as accurately as possible based on physics laws. ${ }^{17-19}$ The other approach is based on synthetic techniques, ${ }^{19-21}$ such as a rain model in which a pebble is randomly dropped in the vessel until it reaches another pebble, after which a Monte Carlo shaking routine is used to increase the packing fraction of the bed. The first method is expected to give realistic packing fractions and porosity profiles, while the second class of methods may not.

This report presents a new method, developed and employed at the INEEL, that belongs to the second class. First, a large collection of random points is generated, about 100,000 times the number of pebbles that would fit within the vessel. Then, starting at the bottom of the vessel, each random point is checked as to whether it is suitable as a pebble center coordinate. If a pebble at that coordinate would overlap with other pebbles or structures in the core, it is rejected; otherwise, it is accepted, and a pebble is located at that position. Besides some problems that need to be avoided, such as integer overflows, this algorithm is extremely simple to program. Furthermore, overlap of pebbles is easily avoided, whereas for the rigorous methods such avoidance is much more difficult. For example, in a packed bed of 5-cm pebbles within a nuclear reactor, it is reported ${ }^{18}$ that the average overlap for the rigorous method is $1 \%$ with a maximum of $20 \%$. However, as may be expected from the discussion above, no physics are simulated in our model, and the resultant pebble-bed packing does not necessarily reflect all characteristics of a real packed bed. Nevertheless, this method was incorporated into the PEBDAN code.

Some results are shown in Figure 11, which displays a comparison with experiments performed by Benenati and Brosilow ${ }^{22}$ in the early sixties. Although the calculations reflect the main trends in radial porosity, there are a few remarkable differences. First of all, the peaks in the calculated porosity profile are not as pronounced as in the measurements. Second, in the calculations, the porosity profile reaches its average value after only two oscillations, while in the measurements at least four oscillations are clearly visible. Third, the average porosity in the calculations $(0.40)$ seems to be larger than the experimental value (0.38). In the algorithm, the density of the packed bed can be increased by generating more random points or by allowing a small overlap between pebbles (as is usually the case in algorithms of the first 
$\operatorname{kind}^{18}$ ). The first option is not very practical, as more random points deteriorate the simplicity and performance of the algorithm, while the second option is unrealistic. [Note: Since completion of the PEBDAN code, we have developed an enhanced version of the rigorous method that produces the correct packing fraction and boundary density fluctuations while suffering very little overlap (of the order of microns at pebble surfaces). This latter method is being verified and corrected for an overly simple treatment of friction between pebbles before incorporation into PEBDAN.]

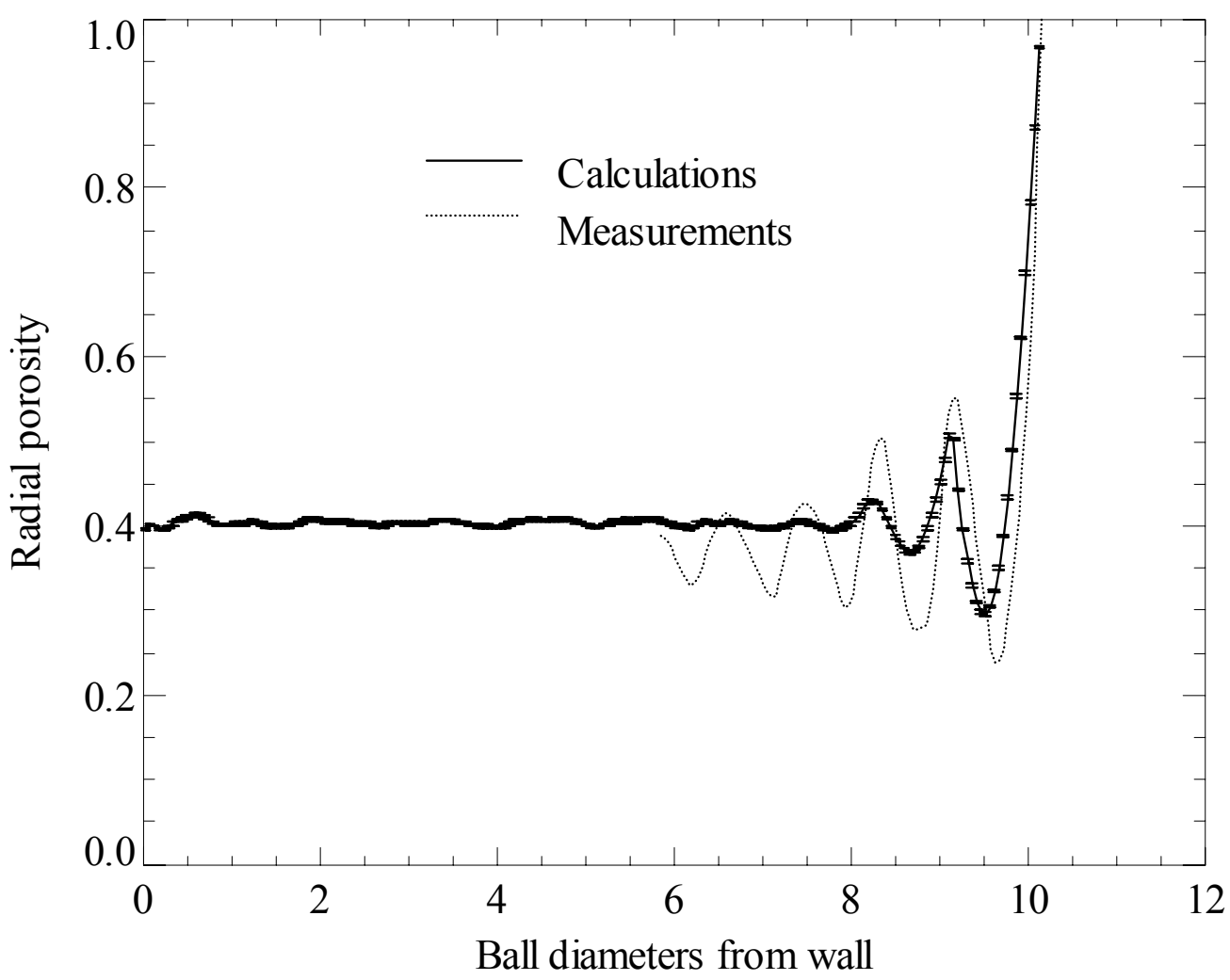

Figure 11. Measured and calculated radial porosity profiles in a cylinder with radius 10.15 times the pebble diameter $(0.080 \mathrm{inch})$. The measurements were read from a plot in the literature ${ }^{22 \text {, case } 2}$ and interpolated with a spline fit to get a fluent curve. Clearly, the calculated peaks in the profile are not as pronounced as in the experimental curve.

In Figure 12, comparison is made with a packed bed of 5-cm pebbles in a nuclear reactor core with a diameter of $3.75 \mathrm{~m} .{ }^{18}$ Again, the peaks in our model are less pronounced, and the porosity is too high. However, two remarks can be made. First, in Reference 18, the pebbles overlap on average with 1\%, which is not the case in our model. Second, compared with the data in Figure 11, the peaks in Reference 18 seem a bit too high. Despite some shortcomings in our model, the results were used to calculate Dancoff factors for the 600-MW Next Generation Nuclear Plant in development at INEEL. 

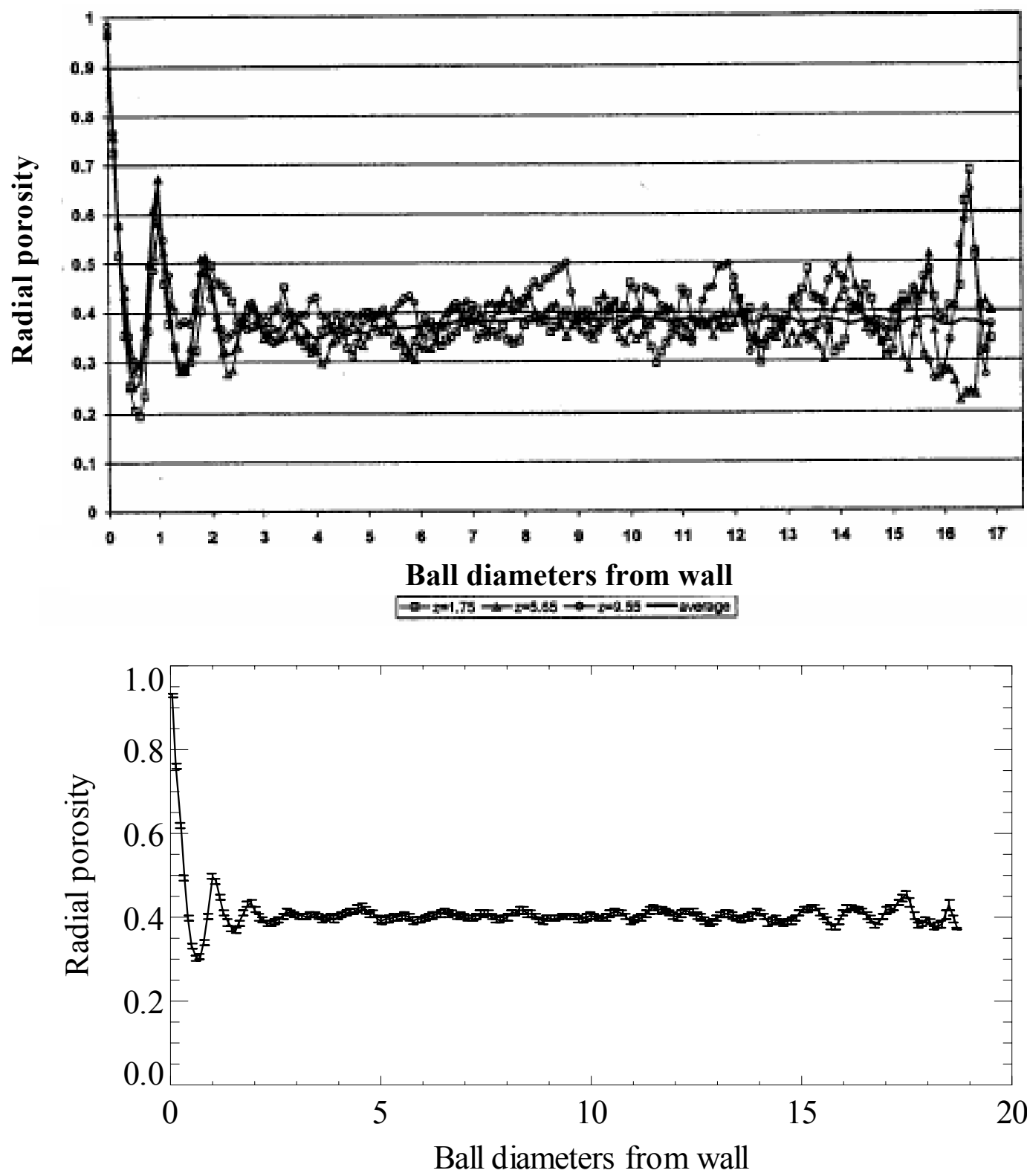

Figure 12. Radial porosity profile of a pebble bed reactor with a diameter of $3.75 \mathrm{~m}$ and a ball diameter of $10 \mathrm{~cm}$, calculated with a discrete element method (Reference 18) and with the algorithm discussed in this paper. As with the measurements in Figure 11, the oscillations calculated here are much less pronounced than the literature values (upper curve).

\subsection{Calculation of Dancoff Factors}

Once the pebble coordinates are known, Dancoff factors are calculated by PEBDAN using a raytracing method. First, a starting coordinate is uniformly sampled in an arbitrary fuel pebble in the core (the core can also contain moderator pebbles, but these are discarded as starting points for the ray-tracing method). Subsequently, a direction is uniformly sampled, and the contribution to the intra-pebble Dancoff factor is determined by calculating the probability that the neutron will interact with a fuel kernel in the same pebble. This is done using Lane's method [Equation (4)] with the upper limit of the integral replaced by the distance the neutron travels in the starting pebble. Subsequently, all pebbles that the ray intersects are stored and ordered with ascending distance to the starting pebble. During the ray tracing 
process, each trajectory through a pebble (either through the moderator zone or through the fuel zone) reduces the weight of the neutron, while each trajectory through the fuel zone of a pebble contributes to the inter-pebble Dancoff factor [again using Lane's method, Equation (4)]. The intra-pebble Dancoff factor should not depend on the starting position in the core, and should virtually be equal to that calculated with Bende's method (Figure 2), while the inter-pebble Dancoff factor will depend on position, as neutrons originating in pebbles in the outer regions of the core experience a larger escape probability.

Besides the intra- and inter-pebble Dancoff factor, the pebble-pebble Dancoff factor is also calculated. The pebble-pebble Dancoff factor is defined as the probability for a neutron that leaves the fuel zone of a pebble to enter another fuel zone without any interaction in between. Actually, it is this Dancoff factor that is needed by the MICROX-2 code in use at INEEL.

In Figure 13, we show the pebble-pebble Dancoff factor as a function of height and radial position in the packed bed of the Next Generation Nuclear Reactor under development at INEEL. Clearly, the Dancoff factor to be input to the MICROX-2 code decreases several tens of percents along the boundaries of the reactor core. The center values $(\approx 0.46)$ are slightly larger than the average value according to Bende's model (0.4436). It must be noted that heretofore, in comparisons between MICROX-2 and other methods (primarily MCNP) conducted by the INEEL NGNP team and their collaborators, this spatial dependence of the pebble-pebble Dancoff factor was never taken into account, and the observed discrepancies remain largely unexplained. This new method should be incorporated into future comparisons. In total, the reactor core volume fraction along the boundaries having a significantly lower Dancoff factor equals about $15 \%$. The influence of this effect on the eigenvalue of the system is something to be investigated in the future.
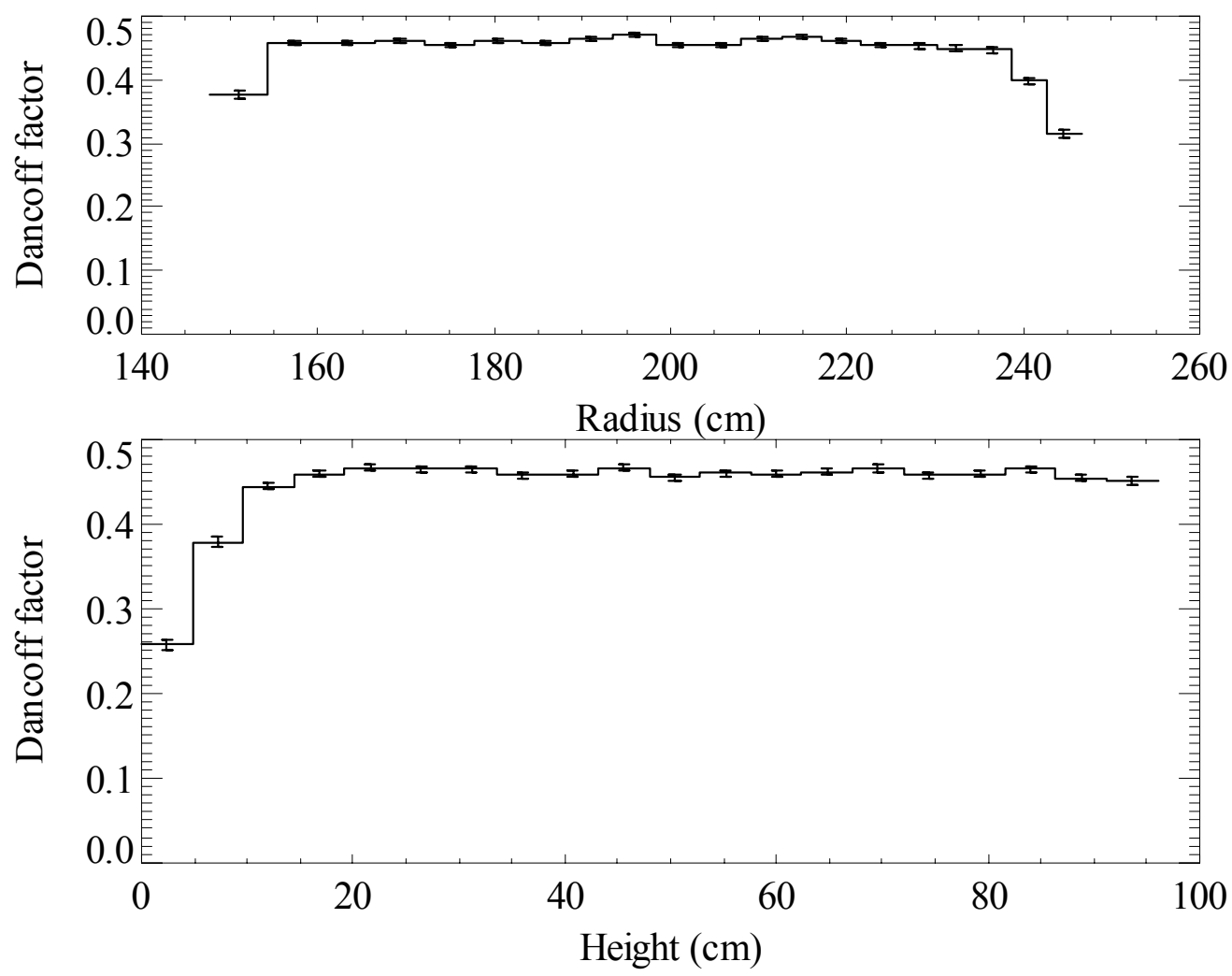

Figure 13. Pebble-Pebble Dancoff factor for the NGNP as a function of radial position (top figure) and height (with a reflective boundary at $96 \mathrm{~cm}$ from the bottom). The radial inner reflector has a radius of $147.8 \mathrm{~cm}$, while the outer reflector starts at $246.6 \mathrm{~cm}$. Clearly, due to leakage, the Dancoff factor decreases several tens of percents at the radial and axial boundaries. 


\section{CONCLUSIONS}

Infinite-medium Dancoff factors, calculated according to four methods reported in the literature, are compared with a dual-particle model developed in this work and reported for the first time. The latter method integrates the Dancoff factor contribution over the surfaces of the two kernels involved and accounts for the stochastic distribution of other kernels in between, while the other methods use either infinitely small kernels (Lane ${ }^{9} /$ Janssen $^{14}$ ), or finite kernels with some (minor) assumptions regarding their stochastic distribution $\left(\right.$ Bende $\left.^{5}\right)$.

The differences between our method and that of Lane are only a few percents. The latter gives remarkably good results at high packing fraction (large particles at high density), while the suggested correction for coated particles worsens the results in this region considerably. Janssen's method, which is very similar to that of Lane, gives deviations between 2 and 3\%, while Bende's method, based on transmission probabilities in spherical geometry, underestimates Dancoff factors at low packing, and overestimates them at high packing. For many practical cases, however, deviations are within one percent.

The intra-pebble Dancoff factor for a single pebble is calculated using a Monte Carlo program called INTRAPEB. The resultant Dancoff factor depends strongly on the radial position within the pebble; it decreases sharply toward the edge of the pebble, while the escape probability shows the opposite tendency. The Dancoff factor for a pebble with a cubic kernel packing arrangement is about $10 \%$ larger than for the same pebble with a stochastic packing of the kernels. The angular distribution at the boundary between the fuel zone and the moderator zone is very similar to a cosine distribution, while at the outer boundary of the moderator, the angular distribution is much more forwardly peaked. This means that the assumption of a cosine angular distribution that is usually applied in analytical methods is not valid at the outer boundary of the pebble.

A program named PEBDAN was written that calculates the pebble coordinates in a stochastically packed bed and the pebble-pebble Dancoff factor as a function of the radial and axial positions in the reactor core. Along the radial and axial boundaries, this Dancoff factor decreases several tens of percents due to enhanced neutron leakage. 


\section{REFERENCES}

S. Feher, J. E. Hoogenboom, P. F. A. De Leege, and J. Valko, "Monte-Carlo Calculation of Dancoff Factors in Irregular Geometries," Nucl. Sci. Eng., 117, 227-238, 1994.

J. R. Knight, Superdan Computer Programs for Calculating the Dancoff Factor of Spheres, Cylinders and Slabs, ORNL/NUREG/CSD-TM-2, Oak Ridge National Laboratory, March 1978.

SCALE: A Modular Code System for Performing Standardized Computer Analyses for Licensing Evaluation, Vols. I-III, NUREG/CR-0200, Rev. 4 (ORNL/NUREG/CSD-2/R4), April 1995. Available from the Radiation Shielding Information Center at Oak Ridge National Laboratory as CCC-545.

E. Teuchert, "Resonanzabsorption in einer Zweifach Heterogenen Anordnung Kugelförmiger Brennelemente," Nucleonik, Vol. 11, pp. 68-72, 1967.

E. E. Bende, A. H. Hogenbirk, J. L. Kloosterman, and H. Van Dam, “Analytical Calculation of the Average Dancoff Factor for a Fuel Kernel in a Pebble Bed High-Temperature Reactor, Nucl. Sci. Eng., Vol. 113, pp. 147-162, 1999.

K. M. Case, F. de Hoffmann, and G. Placzek, Introduction to the Theory of Neutron Diffusion, Vol I, Los Alamos Scientific Laboratory, 1953.

R. M. Westfall, "Cosine Current Transmission Probabilities for Spherical Cells," Trans. Am. Nucl. Soc., Vol. 18, pp. 147-148, 1974.

MICROX-2, Code System to Create Broad-Group Cross Sections with Resonance Interference and SelfShielding from Fine Group and Point Wise Cross Sections, Paul Scherrer Institute, PSR-374, January 1999.

R. K. Lane, L. W. Nordheim, and J. B. Samson, "Resonance Absorption in Materials with Grain Structure,” Nucl. Sci. Eng., Vol. 14, pp. 390-396, 1962.

T. K. Kim, W. S. Yang, T. A. Taiwo, and M. A. Smith, "Preliminary Assessment of Lattice Physics Capabilities for VHTR Analysis," ANS Winter Meeting, November 14-18, Washington DC, U.S., 2004.

J. J. Duderstadt and L. J. Hamilton, Nuclear Reactor Analysis, New York: John Wiley \& Sons, 1986.

G. I. Bell and S. Glasstone, Nuclear Reactor Theory, New York: Van Nostrand Reinhold Company, 1970.

W. J. M. de Kruijf and J. L. Kloosterman, "On the Average Chord Length in Reactor Physics," Ann. Nucl. Ener., Vol. 30, pp. 549-553, 2003.

A. J. Janssen, "Enkele Opmerkingen Over de Dancoff Factor," FYS-LWR-89-11, Netherlands Energy Research Foundation (ECN), January 1990.

S. Kato, S. Yoshimuta, T. Hasumi, K. Sato, K. Sawa, S. Suzuki, H. Mogi, S. Shiozawa, and T. Tanaka, "Fabrication of HTTR First Loading Fuel," IAEA-TECDOC--1210, pp. 187-199, 1998.

C. Tang, Y. Tang, J. Zhu, Y. Zou, J. Li, and X. Ni, "Design and Manufacture of the Fuel Element for the 10 MW High Temperature Gas-Cooled Reactor," Nucl. Eng. Des., Vol. 218, pp. 91-102, 2002.

P. L. Spedding, and R. M. Spencer,"Simulation of Packing Density and Liquid Flow in Fixed Beds," Computers Chem. Engng., Vol. 19, No. 1, pp. 43-73, 1995.

C. G. du Toit, "The Numerical Determination of the Variation in the Porosity of the Pebble-Bed Core," Proc. HTR-TN 2002, April 22-24, Petten, The Netherlands, 2002. 
H. Freund, T. Zeiser, F. Huber, E. Klemm, G. Brenner, F. durst, and G. Enig, "Numerical Simulations of Single Phase Reacting Flows in Randomly Packed Fixed-Bed Reactors and Experimental Validation," Chemical Engineering Science, Vol. 58, pp. 903-910, 2003.

W. Soppe, "Computer Simulation of Random Packings of Hard Spheres," Powder Technology, Vol. 62, pp. 189-196, 1990.

R. Julien, A. Pavlovitch, and P. Meakin, "Random Packings of Spheres Built with Sequential Models," J. Phys. A: Math. Gen., Vol. 25, pp. 4103-4113, 1992.

R. F. Benenati and C. B. Brosilow, "Void Fraction Distribution in Beds of Spheres," A.I.Ch.E. Journal, Vol. 8, No. 3, pp. 359-361, 1962. 